Pacific Journal of Mathematics

NONCOINCIDENCE INDEX, FREE GROUP ACTIONS, AND
THE FIXED POINT PROPERTY FOR MANIFOLDS 


\title{
NONCOINCIDENCE INDEX, FREE GROUP ACTIONS, AND THE FIXED POINT PROPERTY FOR MANIFOLDS
}

\author{
Michael Hoffman
}

\begin{abstract}
Let $M$ be a compact oriented connected topological manifold. We show that if the Euler characteristic $\chi(M) \neq 0$ and $M$ admits no degree zero self-maps without fixed points, then there is a finite number $r$ such that any set of $r$ or more fixed-point-free self-maps of $M$ has a coincidence (i.e. for two of the maps $f$ and $g$ there exists $x \in M$ so that $f(x)=g(x))$. We call $r$ the noncoincidence index of $M$. More generally, for any manifold $M$ with $\chi(M) \neq 0$ there is a finite number $r$ (called the restricted noncoincidence index of $M$ ) so that any set of $r$ or more fixed-point-free nonzero degree self-maps of $M$ has a coincidence. We investigate how these indices change as one passes from a space to its orbit space under a free action. We compute the restricted noncoincidence index for certain products and for the homogeneous spaces $S U_{n} / K, K$ a closed connected subgroup of maximal rank; in some cases these computations also give the noncoincidence index of the space.
\end{abstract}

1. Introduction. Let $M$ be a connected topological manifold. In an earlier paper [10] the author introduced the noncoincidence index, defined as $1+m$ if the maximum cardinality $m$ of a set of noncoincident fixed-point-free self-maps of $M$ is finite, and as $\infty$ otherwise. We shall denote the noncoincidence index of $M$ by $N I(M)$. Two immediate consequences of the definition are:

1. If $N I(M)<\infty$, then any self-map of $M$ has a periodic point of period at most $N I(M)$;

2. If a group $G$ acts freely on $M$, then $|G| \leq N I(M)$.

In [10] the author established some sufficient conditions for $N I(M)$ to be $\infty$ and did explicit calculations of $N I(M)$ in some cases where $N I(M)$ is finite. The first major result of this paper characterizes the compact oriented manifolds of finite noncoincidence index.

THEOREM 1.1. If $M$ is a compact oriented manifold, then $N I(M)<$ $\infty$ if and only if $\chi(M) \neq 0$ and $M$ admits no fixed-point-free self-maps of degree zero. In this case

$$
N I(M) \leq \beta_{1}^{2}+\beta_{2}^{2}+\cdots+\beta_{n}^{2},
$$

where $\beta_{1}, \beta_{2}, \ldots, \beta_{n}$ are the Betti numbers of $M$. 
REMARK. The bound (1) may be compared to similar bounds for periodic points and free group actions. If $X$ is a compact polyhedron with $\chi(X) \neq 0$ and $f$ is a map from $X$ to itself that induces an automorphism of $H^{*}(X ; \mathbf{Q})$, then $f$ has a periodic point of period at most

$$
\max \left(\sum_{i \text { even }} \beta_{i}, \sum_{i \text { odd }} \beta_{i}\right),
$$

where $\beta_{i}$ is the $i$ th Betti number of $X$ (see [3] or [8]). Also, any group acting freely on such a polyhedron $X$ has order at most $|\chi(X)|$, since it must divide $\chi(X)$.

It follows immediately from the definition that $N I(M)=1$ if and only if $M$ has the fixed point property. If $N I(M)$ is finite but not 1 , there may be a free action by a group $G$ so that the orbit space $M / G$ has the fixed point property. In $\S 3$ we prove some results of this type: the simplest is the following one.

THEOREM 1.2. Let $M$ be a compact oriented simply connected manifold, and suppose a finite group $G$ acts freely on $M$. Then $N I(M / G) \leq$ $N I(M) /|G|$. (In particular, $M / G$ has the fixed point property if $|G|=$ $N I(M)$.

The usefulness of the noncoincidence index is limited by the fact that $N I(M)=\infty$ for so many manifolds $M$. For example, the noncoincidence index of a product is $\infty$ unless both factors have the fixed point property. In $\S 4$ we discuss the restricted noncoincidence index, which is always equal to the noncoincidence index when the latter is finite, but which is finite for a larger class of manifolds. We have the following result about products.

THEOREM 1.3. Let $M$ be a product of even-dimensional spheres, complex projective spaces, and quaternionic projective spaces $\mathbf{H} P^{n}$ with $n$ even. Then the restricted noncoincidence index of $M$ is the product of the noncoincidence indices of its indecomposable factors.

In $\S 5$ we compute the restricted noncoincidence of any generalized complex flag manifold (i.e. homogeneous space $G / K$, where $G$ is a compact simple Lie group of type $A_{n}$ and $K$ is a closed connected subgroup of maximal rank). This can be improved to a computation of the noncoincidence index for some classes of such flag manifolds. In particular, we prove the following result. 
THEOREM 1.4. Let $M$ be a generalized complex flag manifold: then $M$ is homeomorphic to $S U_{n} / K$, where $K$ is a subgroup of the form $S U_{n} \cap U_{n_{1}} \times \cdots \times U_{n_{k}}$ with $n_{1} \leq n_{2} \leq \cdots \leq n_{k}, n_{1}+\cdots+n_{k}=n$. Suppose $n$ is even or no more than one odd number occurs in the sequence $n_{1}, n_{2}, \ldots, n_{k}$ with odd multiplicity. Then there is a free action by a finite group $G$ on $M$ so that $M / G$ has the fixed point property provided one of the following conditions holds:

1. $k=2$ and $n_{1} \leq 3$ or $n_{2} \geq 2 n_{1}^{2}-n_{1}-1$;

2. $k=3, n_{1}=1$, and $n_{3} \geq 2 n_{2}^{2}-1$;

3. $n_{1}=n_{2}=\cdots=n_{k-1}=1$.

2. The noncoincidence index. In this section we establish some general results on the noncoincidence index of a compact oriented $n$ manifold $M$. We also prove some results helpful in computing the noncoincidence index from information about the automorphisms of $H^{*}(M ; \mathbf{Q})$.

For any self-map $f$ of $M$, the diagram

$$
M \stackrel{i_{f}}{\longrightarrow} M \times M \stackrel{\mathrm{pr}_{1}}{\longrightarrow} M,
$$

where $i_{f}(x)=(x, f(x))$ and $\mathrm{pr}_{1}$ is projection onto the first factor, is a microbundle in the sense of Milnor [15]. In fact it is the pullback microbundle $f^{*} \mathbf{t}_{M}$, where $\mathbf{t}_{M}$ is the tangent microbundle of $M$. The 'zero section' of $f^{*} \mathbf{t}_{M}$ (i.e., the image of $i_{f}$ ) is gr $f$, the graph of $f$. By a theorem of Kister [13], any sufficiently small neighborhood of gr $f$ in $M \times M$ is an $\mathbf{R}^{n}$-bundle over $M$ (where $\mathbf{R}^{n}$ is thought of as having a distinguished origin but no linear structure). We use this fact in proving the next result.

THEOREM 2.1. For any sufficiently small neighborhood $U$ of $\operatorname{gr} f$ in $M \times M, \operatorname{pr}_{1}: U-\operatorname{gr} f \rightarrow M$ is an $\left(\mathbf{R}^{n}-0\right)$-bundle and the obstruction to sectioning it is $\operatorname{deg} f \chi(M)$.

Proof. Take $U$ sufficiently small so that $U \stackrel{\mathrm{pr}_{1}}{\longrightarrow} M$ is an $\mathbf{R}^{n}$-bundle. Since $M$ is oriented, so is any bundle realization of $\mathbf{t}_{M}$ or $f^{*} \mathbf{t}_{M}$. Then the obstruction to sectioning $U-\operatorname{gr} f \stackrel{\mathrm{pr}_{1}}{\longrightarrow} M$ is an element of $H^{n}(M ; \mathbf{Z})$. For $f=\mathrm{id}$, this obstruction is the Euler class $e(M)$ : by naturality, the obstruction in the general case is $f^{*} e(M)$ (cf. [17]). Since $\left\langle f^{*} e(M),[M]\right\rangle=\operatorname{deg} f \chi(M)$, the conclusion follows.

THEOREM 2.2. If $M$ admits a fixed-point-free self-map of degree zero, then $N I(M)=\infty$. 
Proof. Let $f: M \rightarrow M$ be a fixed-point-free map with $\operatorname{deg} f=0$. Then gr $f \cap \Delta(M)=\varnothing$, where $\Delta(M) \subset M \times M$ is the diagonal. Take a neighborhood $U_{1}$ of gr $f$ small enough so that $U_{1} \cap \Delta(M)=\varnothing$ and $U_{1} \stackrel{\mathrm{pr}_{1}}{\longrightarrow} M$ is an $\mathbf{R}^{n}$-bundle: then the previous result implies that there is a section of $U_{1}-\operatorname{gr} f \stackrel{\mathrm{pr}_{1}}{\longrightarrow} M$. Such a section gives a map $f_{1}: M \rightarrow M$ which is fixed-point-free and noncoincident with $f$. Now repeat this procedure: take a neighborhood $U_{2} \subset U_{1}$ of $\operatorname{gr} f$ with $U_{2} \cap$ gr $f_{1}=\varnothing$. Sectioning $U_{2}-\operatorname{gr} f \stackrel{\mathrm{pr}_{1}}{\longrightarrow} M$ gives a map $f_{2}: M \rightarrow M$ which is fixed-point-free and noncoincident with $f$ and $f_{1}$. In this way we get $N I(M) \geq k$ for any $k$.

REMARK. A similar argument (taking neighborhoods of $\Delta(M)$ ) shows that $N I(M)=\infty$ when $\chi(M)=0$. This is proved without assuming orientability in [10].

Let $L Z(M)=\left\{f^{*}: H^{*}(M ; \mathbf{Q}) \rightarrow H^{*}(M ; \mathbf{Q}) \mid f\right.$ is fixed-point-free $\}$. (This is a slight modification of the definition used in [10].) By the Lefschetz fixed point theorem $L(\phi)=0$ for any $\phi \in L Z(M)$, where $L(\phi)$ is the Lefschetz number

$$
L(\phi)=\sum_{i \geq 0}(-1)^{i} \operatorname{Tr}\left(\phi_{i}: H^{i}(M ; \mathbf{Q}) \rightarrow H^{i}(M ; \mathbf{Q})\right) .
$$

If $L Z(M)$ consists of automorphisms of $H^{*}(M ; \mathbf{Q})$ (i.e. endomorphisms of nonzero degree) and $\chi(M) \neq 0$, we call $M L$-rigid. By Theorem 2.2 (and the subsequent remark), $N I(M)=\infty$ unless $M$ is L-rigid.

We call automorphisms $\phi, \psi$ of $H^{*}(M ; \mathbf{Q})$ compatible if $L\left(\phi \psi^{-1}\right)=$ 0 . (Note that $L(\mu)=\operatorname{deg} \mu L\left(\mu^{-1}\right)$, so the relation is symmetric; it need not be reflexive or transitive.) If $M$ is $L$-rigid, every element of $L Z(M)$ is compatible with the identity. From [10] we have the following result, which is proved by using the Lefschetz coincidence theorem.

THeOREM 2.3. If $M$ is L-rigid and $N I(M) \geq k$, then $L Z(M)^{\prime}=$ $L Z(M) \cup\{\mathrm{id}\}$ contains a set of $k$ pairwise compatible elements that includes the identity.

Theorem 2.3 has an immediate corollary in the case where $H^{*}(M ; \mathbf{Q})$ is a truncated polynomial algebra on an even-dimensional generator. Suppose $H^{*}(M ; \mathbf{Q})$ is the polynomial algebra on $x \in H^{2 d}(M ; \mathbf{Q})$ modulo the relation $x^{h+1}=0$ : we call $h$ the height of $x$. Then any endomorphism $\phi$ of $H^{*}(M ; \mathbf{Q})$ has the form $\phi(x)=a x$, and by direct 
computation its Lefschetz number is

$$
L(\phi)=1+a+a^{2}+\cdots+a^{h} .
$$

If we denote the right-hand side by $P_{h}(a)$, then $P_{h}(1)=h+1$ and

$$
P_{h}(a)=\frac{1-a^{h+1}}{1-a}
$$

if $a \neq 1$. It follows that $P_{h}(a)=0$ if and only if $h$ is odd and $a=-1$.

The rational cohomology algebras of an even-dimensional sphere $S^{2 n}$, complex projective space $\mathbf{C} P^{n}$, and quaternionic projective space $\mathbf{H} P^{n}$ are truncated polynomial algebras on even-dimensional generators of heights $2, n$, and $n$ respectively. Then $N I\left(S^{2 n}\right) \leq 2$ by Theorem 2.3, and in fact $N I\left(S^{2 n}\right)=2$ since the antipodal map is fixed-pointfree. Similarly $N I\left(\mathbf{C} P^{n}\right)=2$ if $n$ is odd, and we have $N I\left(\mathbf{C} P^{n}\right)=1$ if $n$ is even. The case for quaternionic projective space is a bit more complicated: we have $N I\left(\mathbf{H} P^{n}\right)=1$, for $n$ even, but also $N I\left(\mathbf{H} P^{n}\right)=1$ for odd $n \neq 1$. This is because there is no self-map of $\mathbf{H} P^{n}$ which induces the endomorphism of $H^{*}\left(\mathbf{H} P^{n} ; \mathbf{Z}\right)$ that sends the generator $x$ to $-x$ for odd $n \neq 1$, as can be seen from mod 3 Steenrod operations [2]. We shall return to these examples in $\S 4$.

The next result completes the proof of Theorem 1.1 by putting an upper bound on the noncoincidence index of an $L$-rigid manifold.

THEOREM 2.4. If $M$ is L-rigid, then $N I(M) \leq \beta_{1}^{2}+\beta_{2}^{2}+\cdots+\beta_{n}^{2}$, where $\beta_{1}, \beta_{2}, \ldots, \beta_{n}$ are the Betti numbers of $M$.

Proof. As in [1] we can find a class $\bar{u} \in H^{n}(M \times M ; \mathbf{Q})$ so that $d^{*}(\bar{u}) \in H^{n}(M ; \mathbf{Q})$ is the Euler class of $M$, i.e.

$$
\left\langle d^{*}(\bar{u}),[M]\right\rangle=\chi(M),
$$

where $d: M \rightarrow M \times M$ is the diagonal map. Further, if we choose a basis $p_{1}, p_{2}, \ldots, p_{k}$ for $H^{*}(M ; \mathbf{Q})$ and let $q_{1}, q_{2}, \ldots, q_{k}$ be a dual basis (so $\left\langle p_{i} \cup q_{j},[M]\right\rangle=\delta_{i j}$ ), then

$$
\bar{u}=\sum_{i=1}^{k}(-1)^{\operatorname{dim} p_{i}} p_{i} \times q_{i} .
$$

Then for any endomorphism $\phi$ of $H^{*}(M ; \mathbf{Q})$, the element

$$
(\phi \times \mathrm{id})(\bar{u})=\sum_{i=1}^{k}(-1)^{\operatorname{dim} p_{i}} \phi\left(p_{i}\right) \times q_{i}
$$

has the property $\left\langle d^{*}((\phi \times \mathrm{id})(\bar{u})),[M]\right\rangle=L(\phi)(\mathrm{cf} .[1])$. 
Now suppose $N I(M)>\beta_{1}^{2}+\beta_{2}^{2}+\cdots+\beta_{n}^{2}=\operatorname{rank} H^{n}(M \times M ; \mathbf{Q})$. Then there is a set $S=\left\{\phi_{0}, \phi_{1}, \ldots, \phi_{r}\right\}$ of pairwise compatible elements of $L Z(M)$, where $\phi_{0}=\mathrm{id}$ and $r=\operatorname{rank} H^{n}(M \times M ; \mathbf{Q})$. The set $\{(\phi \times \mathrm{id})(\bar{u}) \mid \phi \in S\}$ is linearly dependent: let

$$
c_{0} \bar{u}+c_{1}\left(\phi_{1} \times \mathrm{id}\right)(\bar{u})+\cdots+c_{r}\left(\phi_{r} \times \mathrm{id}\right)(\bar{u})=0
$$

be a relation of linear dependence. If $c_{0} \neq 0$, then we can apply the operator $\left\langle d^{*}(\cdot),[M]\right\rangle$ to (1) to obtain $c_{0} \chi(M)=0$, contradicting the hypothesis. Otherwise, first choose $c_{i} \neq 0$ and apply $\phi_{i}^{-1} \times$ id to (1).

REMARK. The argument used here is essentially that of Fuller [3].

If $S$ is a set of pairwise compatible automorphisms of $H^{*}(M ; \mathbf{Q})$ that includes the identity, we call $S$ a compatible set for $M$. In view of Theorem 2.3, we can bound $N I(M)$ by bounding the cardinality of compatible sets for $M$. The following results will be used in $\S \S 4-5$.

Proposition 2.5. Let $S$ be a compatible set for $M$ contained in a subgroup $G$ of Aut $H^{*}(M ; \mathbf{Q})$, and suppose $H$ is a subgroup of finite index in $G$ such that $L(h) \neq 0$ for all $h \in H$. Then $|S| \leq[G: H]$.

Proof. Let $S$ be a compatible set for $M$. The conclusion follows if the cosets $H \phi$ and $H \psi$ are distinct for any two distinct elements $\phi, \psi \in S$. Suppose otherwise: then $\phi=h \psi$ for some $h \in H$, and $L(h)=L\left(\phi \psi^{-1}\right)=0$, contradicting the hypothesis.

Proposition 2.6. Suppose $S$ is a compatible set for $M$ contained in a finite subgroup $G$ of Aut $H^{*}(M ; \mathbf{Q})$, and let $R$ be a subset of $G$ such that id $\in R$ and $L\left(\rho^{-1} \sigma\right) \neq 0$ for any $\rho, \sigma \in R$. Then $|S| \leq|G| /|R|$.

Proof. It suffices to show that $\rho S \cap \sigma S=\varnothing$ for distinct elements $\rho$, $\sigma$ of $R$. Suppose otherwise: then there are distinct elements $s_{1}, s_{2}$ of $S$ so that $\rho s_{1}=\sigma s_{2}$. But then $L\left(s_{1} s_{2}^{-1}\right)=L\left(\rho^{-1} \sigma\right) \neq 0$ contradicting the compatibility of $S$.

3. Free group actions and the fixed point property. If $M$ is a simply connected, compact oriented manifold that admits a free action by a finite group $G$, information about the relationship between the noncoincidence index of the orbit space $M / G$ and that of $M$ can be obtained by studying the finite covering $M \rightarrow M / G$. In this section we prove a number of theorems about this situation. 
Suppose now that $G$ is a fintie group that acts freely on $M$, a compact oriented manifold. Then $H^{*}(M / G ; \mathbf{Q})=H^{*}(M ; \mathbf{Q})^{G}$, the subalgebra of $G$-invariants. (Note that $M / G$ is a manifold, but need not be orientable since we are not assuming that the elements of $G$ preserve orientation.) We can assume $\chi(M) \neq 0$, since otherwise $M$ and $M / G$ have noncoincidence index $\infty$. Let $L Z(M)^{\prime}=L Z(M) \cup\{$ id $\}$, and define $L Z(M)^{G}$ to be the set of elements of $L Z(M)^{\prime}$ that preserve $H^{*}(M ; \mathbf{Q})^{G}$.

If $M$ is simply connected, then $M$ is the universal cover of the manifold $M / G$. Given any map $f: M / G \rightarrow M / G$, there is a 'lift' $\tilde{f}: M \rightarrow M$ so that the diagram

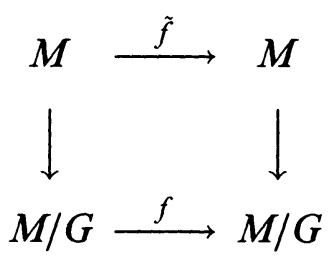

commutes. If $\tilde{f}, \tilde{g}$ are lifts of noncoincident self-maps $f, g$ of $M / G$, then $\tilde{f}(x)$ and $\tilde{g}(x)$ are in different $G$-orbits for every $x \in M$. Applying $H^{*}(\cdot ; \mathbf{Q})$ to the diagram $(1)$, we see that $\tilde{f}^{*}$ takes the subalgebra $H^{*}(M ; \mathbf{Q})^{G}$ into itself. In fact we have the following result.

THEOREM 3.1. Let $M$ be a compact oriented simply connected manifold of nonzero Euler characteristic. If a finite group $G$ acts freely on $M$ and $N I(M / G) \geq k$, then $L Z(M)^{G}$ contains a compatible subset of $k|G|$ elements that includes $G$.

Proof. Let $\left\{f_{1}, f_{2}, \ldots, f_{k}\right\}$ be a set of noncoincident self-maps of $M / G$ with $f_{1}=$ id. For each $f_{i}$ choose a lift $\tilde{f}_{i}: M \rightarrow M$. Then the set $\left\{g \tilde{f}_{i} \mid g \in G, 1 \leq i \leq k\right\}$ has the properties required by the conclusion.

Theorem 1.2 follows immediately from this result and Theorem 2.3. We give two other corollaries: in each $M$ satisfies the hypotheses of Theorem 3.1 and $G$ acts freely on $M$. (Note that $G$ can be regarded as a subset of $L Z(M)^{\prime}$.)

Corollary 3.2. If $G \subset L Z(M)^{\prime}$ is maximal as a set of pairwise compatible elements of $L Z(M)^{G}$, then $M / G$ has the fixed point property.

COROLLARY 3.3. If $L Z(M)^{\prime}$ is a group and $N(G)$ (the normalizer of $G$ in $\left.L Z(M)^{\prime}\right)$ acts freely on $M$, then $N I(M / G)=|N(G) / G|$. 
It is interesting to compare the preceding results to the following one, which does not use the idea of noncoincidence index.

TheOREM 3.4. Suppose $H^{*}(M ; \mathbf{Q})$ is concentrated in even dimensions and a group $G$ of order $\chi(M)$ acts freely on $M$. Then $M / G$ has the fixed point property.

Proof. Since $\chi(M / G)=\chi(M) /|G|=1$, the reduced rational cohomology $\tilde{H}^{*}(M / G ; \mathbf{Q})$ is trivial. But then the Lefschetz fixed point theorem implies $M / G$ has the fixed point property.

Both Theorem 1.2 and Theorem 3.4 can be used to prove that $\mathbf{R} P^{n}$ has the fixed point property for $n$ even, since in that case $N I\left(S^{n}\right)=$ $\chi\left(S^{n}\right)=2$. But Theorem 1.2 shows that the orbit space of $\mathbf{C} P^{n}$, $n \geq 3$ odd, has the fixed point property for any free $\mathbf{Z}_{2}$-action, while Theorem 3.4 does not apply since $\chi\left(\mathbf{C} P^{n}\right)=n+1 \geq 4$.

4. Products and the restricted noncoincidence index. A difficulty with the noncoincidence index is that it is so often infinite. In fact, we have the following result.

Proposition 4.1. Let $M$ be a compact manifold with $N I(M)<\infty$. Then any retract of $M$ (other than $M$ itself) has the fixed point property.

Proof. Suppose $L \subset M$ is a retract admitting a fixed-point-free map $f$. Let $i: L \rightarrow M$ be the inclusion, $r: M \rightarrow L$ a retraction. If $L \neq M$, then ifr: $M \rightarrow M$ is a nonsurjective fixed-point-free map. But then $N I(M)=\infty$ by Theorem 2.2 of [10].

Thus, any product $M \times N$ has noncoincidence index $\infty$ unless both $M$ and $N$ have the fixed point property. If $M$ is a compact oriented manifold, we define the restricted noncoincidence index of $M$, $R N I(M)$, by putting $R N I(M) \geq k+1$ whenever $M$ admits $k$ selfmaps of nonzero degree, each of which is fixed-point-free and no two of which have a coincidence. Evidently $R N I(M) \leq N I(M)$. We have the following results from the proofs of Theorems 2.3, 2.4 and 3.1, respectively.

Proposition 4.2. If $M$ is a compact oriented manifold with $\chi(M) \neq$ 0 and $R N I(M) \geq k$, then $L Z(M)^{\prime}$ contains a compatible set for $M$ with $k$ elements.

Proposition 4.3. For any compact oriented manifold $M, R N I(M)$ $=N I(M)$ whenever the latter is finite. Further, $R N I(M)<\infty$ if and 
only if $\chi(M) \neq 0$, in which case

$$
R N I(M) \leq \beta_{1}^{2}+\beta_{2}^{2}+\cdots+\beta_{n}^{2},
$$

where $\beta_{1}, \beta_{2}, \ldots, \beta_{n}$ are the Betti numbers of $M$.

Proposition 4.4. Let $M$ be a compact oriented simply connected manifold on which a group $G$ acts freely by orientation-preserving homeomorphisms. Then $R N I(M / G) \leq R N I(M) /|G|$.

We note that any group $G$ acting freely on $M$ has order $|G| \leq$ $R N I(M)$, and that, if $\chi(M) \neq 0$, any self-map of $M$ of nonzero degree has a periodic point of period at most $R N I(M)$. Also, $R N I(M)=1$ if and only if $M$ has the fixed point property for maps of nonzero degree.

It is evident that $R N I(M \times N) \geq R N I(M) R N I(N)$ for any two manifolds $M$ and $N$. Equality holds in many cases (Theorem 1.3), but the inequality can be strict even for products of spheres and projective spaces (Proposition 4.7).

For many manifolds $M$, it is possible to classify the automorphisms of $H^{*}(M ; \mathbf{Q})$ and use Proposition 2.5 or 2.6 together with Proposition 4.2 to compute $R N I(M)$. Let $A$ be a graded algebra that is a tensor product of truncated polynomial algebras, with even dimensional generators $x_{1}, \ldots, x_{n}$. Then from [11] we have the following results (ht $x_{i}$ denotes the height of $x_{i}$ ).

THEOREM 4.5. Any automorphism $\phi$ of $A$ has the form $\phi\left(x_{i}\right)=$ $a_{i} x_{\sigma(i)}$, where $\sigma$ is an element of the symmetric group $\Sigma_{n}$ such that $\operatorname{dim} x_{i}=\operatorname{dim} x_{j}$ and ht $x_{i}=\mathrm{ht} x_{j}$ whenever $\sigma(i)=j$.

THEOREM 4.6. Let $\phi$ be the automorphism of $A$ given by $\phi\left(x_{i}\right)=$ $a_{i} x_{\sigma(i)}$. Then

$$
L(\phi)=\prod_{i=1}^{k} P_{h_{i}}\left(\prod_{j \in S_{\imath}} a_{j}\right),
$$

where $\{1,2, \ldots, n\}=S_{1} \cup \cdots \cup S_{k}$ is the partition corresponding to $\sigma$, $h_{i}=\mathrm{ht} x_{j}$ for $j \in S_{i}$, and $P_{h}$ is the polynomial introduced in $\S 2$.

As noted in $\S 2$, the cohomology algebras of even-dimensional spheres and complex and quaternionic projective spaces are truncated polynomial algebras on even-dimensional generators. We can use the algebraic results above to prove Theorem 1.3. 
Proof of 1.3. The hypothesis insures that $H^{*}(M ; \mathbf{Q})$ is a tensor product of truncated polynomial algebras on even-dimensional generators $x_{1}, \ldots, x_{n}$. Suppose first that the $x_{i}$ all have the same height and dimension. Then by Theorem 4.5 any automorphism of $H^{*}(M ; \mathbf{Q})$ can be represented by $\left(a_{1}, \ldots, a_{n} ; \sigma\right)$, where the $a_{i}$ are nonzero rational numbers and $\sigma \in \Sigma_{n}$. In fact, the automorphism group of $H^{*}(M ; \mathbf{Q})$ is the semidirect product $\left(\mathbf{Q}^{*}\right)^{n} \rtimes \Sigma_{n}$, where $\mathbf{Q}^{*}$ is the group of nonzero rational numbers and $\Sigma_{n}$ acts on $\left(\mathbf{Q}^{*}\right)^{n}$ by permuting its factors. Now from Theorem 4.6 it follows that the Lefschetz number of $\left(a_{1}, \ldots, a_{n} ; \sigma\right)$ is always nonzero if the common height of the $x_{i}$ is even, and zero exactly when the product of the $a_{i}$ over some cycle of $\sigma$ is -1 if the height is odd. In the first case $R N I(M)=1$. In the second case there is a subgroup of index $2^{n}$ in $\mathbf{Q}^{*} \rtimes \Sigma_{n}$ given by the elements $\left(a_{1}, \ldots, a_{n} ; \sigma\right)$ with all the $a_{i}$ positive (a complete set of coset representatives is given by those elements with $\sigma=\mathrm{id}$ and $a_{i}= \pm 1$ for each $i$ ); every element of this subgroup has nonzero Lefschetz number. But then we can apply Propositions 2.5 and 4.2 to conclude that $R N I(M) \leq 2^{n}$.

For the general case, let $\{1,2, \ldots, n\}=S_{1} \cup \cdots \cup S_{k}$ be the coarsest partition such that all generators labelled by elements of a set $S_{i}$ have the same dimension and height. Then $H^{*}(M ; \mathbf{Q})=A_{1} \otimes \cdots \otimes A_{k}$, where $A_{i}$ is the subalgebra generated by $\left\{x_{j} \mid j \in S_{i}\right\}$. Theorem 4.4 implies that the automorphisms of $H^{*}(M ; \mathbf{Q})$ leave the subalgebras $A_{i}$ invariant, and the automorphism group of $H^{*}(M ; \mathbf{Q})$ is just the direct product of those for $A_{1}, \ldots, A_{k}$. By the argument of the preceding paragraph, $R N I(M)$ is bounded by the product of the numbers $2^{\left|S_{l}\right|}$ (for each $i$ such that the generators of $A_{i}$ have odd height) and 1 (for each $i$ such that the generators of $A_{i}$ have even height). But (by the remarks following 2.3) this is the product of the noncoincidence indices of the factors of $M$.

Proposition 4.7. $R N I\left(S^{2} \times \mathbf{H} P^{3}\right)=3$.

Proof. By Theorem 4.5, all automorphisms of the cohomology of $S^{2} \times \mathbf{H} P^{3}$ can be written as $\phi \otimes \psi$ for automorphisms $\phi, \psi$ of $H^{*}\left(S^{2} ; \mathbf{Q}\right)$ and $H^{*}\left(\mathbf{H} P^{3} ; \mathbf{Q}\right)$ respectively. Since $L(\phi \otimes \psi)=L(\phi) L(\psi)$ and $L(\psi) \neq$ 0 for every automorphism $\psi$ induced by a self-map of $\mathbf{H} P^{3}$, the elements of $L Z\left(S^{2} \times \mathbf{H} P^{3}\right)$ all have the form $a^{*} \otimes \psi$, where $a$ is the antipodal map of $S^{2}$. For two such maps to be noncoincident it is 
necessary for their Lefschetz coincidence number to be zero, i.e.

$$
\begin{aligned}
0 & =L\left(a^{*} \otimes \phi_{1}, a^{*} \otimes \psi_{2}\right)=-L\left(\left(a^{*} \otimes \psi_{1}\right)^{-1} a^{*} \otimes \psi_{2}\right) \\
& =-L\left(\mathrm{id} \otimes \psi_{1}^{-1} \psi_{2}\right)=-L\left(\psi_{1}^{-1} \psi_{2}\right) .
\end{aligned}
$$

Now the only automorphism of $H^{*}\left(\mathbf{H} P^{3} ; \mathbf{Q}\right)$ with Lefschetz number zero is the one that multiplies the generator by -1 . This is not induced by any self-map of $\mathbf{H} P^{3}$, but it can be obtained as $\psi_{1}^{-1} \psi_{2}$ for $\psi_{1}, \psi_{2}$ induced by self-maps of $\mathbf{H} P^{3}$ : for example, we can choose $\psi_{1}$ to multiply the generator by 360 , and $\psi_{2}$ to multiply it by -360 (see [14]). Then the maps corresponding to $\psi_{1}$ and $\psi_{2}$ have Lefschetz coincidence number zero, and can be deformed to noncoincident maps $f_{1}, f_{2}$ [1], so $a \times f_{1}, a \times f_{2}$ are fixed-point-free noncoincident maps of the product: clearly this is the largest possible such set.

5. Complex flag manifolds. Manifolds of finite noncoincidence index occur 'naturally' as homogeneous spaces of the form $G / K$, where $G$ is a simple compact connected Lie group and $K$ is a closed connected subgroup of maximal rank. The simplest situation is when $K=T$, a maximal torus. Papadima [16], generalizing an earlier result of the author [9], has recently shown that $G / T$ has noncoincidence index equal to the order of the Weyl group $W(G)=N_{G} T / T\left(N_{G} T\right.$ is the normalizer of $T$ in $G$ ). Since $W(G)$ also acts freely on $G / T$, the orbit space $G / N_{G} T$ has the fixed point property by Theorem 1.2. (One can also use Theorem 3.4, since $\chi(G / T)=|W(G)|$ and the rational cohomology of $G / T$ is concentrated in even dimensions.)

It appears that similar results hold if $K$ has maximal rank but is not a torus. But even for the class of homogeneous spaces studied in [10], there were cases where the noncoincidence index was infinite. Here we study the case where $G$ is of type $A_{n}$, where the most results on the cohomology endomorphisms of $G / K$ seem to be available.

We consider homogeneous spaces of the form $S U_{n} / K$, where $K$ is a closed subgroup of maximal rank: it is well-known that $K$ is conjugate to a subgroup of the form $S U_{n} \cap U_{n_{1}} \times U_{n_{2}} \times \cdots \times U_{n_{k}}$, where $n_{1}+\cdots+n_{k}=n$. Henceforth we shall assume without loss of generality that $K=S U_{n} \cap U_{n_{1}} \times \cdots \times U_{n_{k}}$ with $n_{1} \leq n_{2} \leq \cdots \leq n_{k}$. The space $S U_{n} / K$ can be regarded as the space of orthogonal decompositions

$$
\mathbf{C}^{n}=p_{1} \oplus \cdots \oplus p_{k}, \quad \operatorname{dim} p_{i}=n_{i} .
$$

The 'Weyl group' for this situation is $N_{G} K / K$, which is

$$
N_{W(G)} W(K) / W(K):
$$


it can be identified with the group of permutations $\sigma \in \Sigma_{k}$ such that $n_{i}=n_{j}$ whenever $\sigma(i)=j$. This group acts on decompositions (1) by sending $p_{i}$ to $p_{\sigma(i)}$, and this action is evidently free.

The cohomology of $S U_{n} / K$ is generated by elements $x_{i j} \in$ $H^{2 j}\left(S U_{n} / K ; \mathbf{Q}\right), 1 \leq i \leq k, 1 \leq j \leq n_{i}$, subject to the relations

$$
\left(1+x_{11}+\cdots+x_{1 n_{1}}\right) \cdots\left(1+x_{k 1}+\cdots+x_{k n_{k}}\right)=1 \text {. }
$$

The action of the group $N_{G} K / K$ on cohomology is easily described: $\sigma \in N_{G} K / K$ sends $x_{i j}$ to $x_{\sigma(i) j}$. More generally, for any rational number $\lambda \neq 0$ and $\sigma \in N_{G} K / K$ we can define an automorphism $\Phi_{\lambda}^{\sigma}$ of $H^{*}\left(S U_{n} / K ; \mathbf{Q}\right)$ by $\Phi_{\lambda}^{\sigma}\left(x_{i j}\right)=\lambda^{j} x_{\sigma(i) j}$ : we call such an automorphism a permutation automorphism. Recently Shiga and Tezuka [18] have proved that every automorphism of $H^{*}\left(S U_{n} / K\right.$; Q $)$ has this form. In order to find the restricted noncoincidence index of $S U_{n} / K$, we first compute the Lefschetz number of a permutation automorphism.

TheOReM 5.1. Let $\lambda$ be a rational number, $\sigma \in N_{G} K / K$ a permutation, and $\{1,2, \ldots, k\}=S_{1} \cup \cdots \cup S_{r}$ the partition associated to $\sigma$. Then

$$
L\left(\Phi_{\lambda}^{\sigma}\right)=(1-\lambda)\left(1-\lambda^{2}\right) \cdots\left(1-\lambda^{n}\right) \prod_{p=1}^{r} \prod_{j=1}^{n\left(S_{p}\right)} \frac{1}{1-\lambda^{j}\left|S_{p}\right|},
$$

where $n\left(S_{p}\right)$ is $n_{i}$ for any (and thus every) $i \in S_{p}$.

Proof. This calculation is a straightforward generalization of those done in [9] and [10]. First note $S U_{n} / K$ is homeomorphic to $U_{n} / K^{\prime}$, where $K^{\prime}=U_{n_{1}} \times \cdots \times U_{n_{k}}$, so we have a fibration

$$
S U_{n} / K \rightarrow B U_{n_{1}} \times \cdots \times B U_{n_{k}} \rightarrow B U_{n} .
$$

Now let

$$
P=H^{*}\left(B U_{n_{1}} \times \cdots \times B U_{n_{k}} ; \mathbf{Q}\right)=\mathbf{Q}\left[x_{i j} \mid 1 \leq i \leq k, 1 \leq j \leq n_{i}\right],
$$

where $x_{i j}$ is in dimension $2 j$. Then $N_{G} K / K$ acts on $P$ by sending $x_{i j}$ to $x_{\sigma(i) j}$, and we have an invariant decomposition

$$
P=\bigotimes_{p=1}^{r} \bigotimes_{j=1}^{n\left(S_{p}\right)} A_{p j}
$$

where $A_{p j}=\mathbf{Q}\left[x_{i j} \mid i \in S_{p}\right]$. Now

$$
\sum_{i \geq 0} t^{i} \operatorname{Tr}\left(\sigma:\left(A_{p j}\right)_{2 i} \rightarrow\left(A_{p j}\right)_{2 i}\right)=\frac{1}{1-t^{j}\left|S_{p}\right|},
$$


so the multiplicative property of trace on tensor products gives

$$
\sum_{i \geq 0} t^{i} \operatorname{Tr}\left(\sigma: P_{2 i} \rightarrow P_{2 i}\right)=\prod_{p=1}^{r} \prod_{j=1}^{n\left(S_{p}\right)} \frac{1}{1-t^{j\left|S_{p}\right|}} .
$$

Now the spectral sequence of the fibration (2) collapses for degree reasons, so

$$
P=H^{*}\left(S U_{n} / K ; \mathbf{Q}\right) \otimes H^{*}\left(B U_{n} ; \mathbf{Q}\right)
$$

additively. Together with (3) and the fact

$$
\sum_{i \geq 0} t^{i} \operatorname{dim} H^{2 i}\left(B U_{n} ; \mathbf{Q}\right)=\frac{1}{(1-t) \cdots\left(1-t^{n}\right)}
$$

this implies

$$
\begin{gathered}
\sum_{i \geq 0} t^{i} \operatorname{Tr}\left(\Phi_{1}^{\sigma}: H^{2 i}\left(S U_{n} / K ; \mathbf{Q}\right) \rightarrow H^{2 i}\left(S U_{n} / K ; \mathbf{Q}\right)\right) \\
=(1-t) \cdots\left(1-t^{n}\right) \prod_{p=1}^{r} \prod_{j=1}^{n\left(S_{p}\right)} \frac{1}{1-t^{j\left|S_{p}\right|}}
\end{gathered}
$$

from which the conclusion follows by putting $t=\lambda$.

It will be convenient to introduce some further notation. For each positive integer $q$, let $M_{q}=\left\{i \mid n_{i}=q\right\}$ and $m_{q}=\left|M_{q}\right|$. We can now give the restricted noncoincidence index of $S U_{n} / K$ as follows.

THEOREM 5.2. Let $M=S U_{n} / K$ be a complex flag manifold. Then $R N I(M)= \begin{cases}\left|N_{G} K / K\right|, & \text { if at most one of } m_{1}, m_{3}, m_{5}, \ldots \text { is odd } \\ 2\left|N_{G} K / K\right|, & \text { otherwise. }\end{cases}$

Proof. First we use 5.1 to determine the automorphisms $\Phi_{\lambda}^{\sigma}$ for which $L\left(\Phi_{\lambda}^{\sigma}\right)=0$. Evidently this only happens if $\lambda= \pm 1$, so it follows at once that $R N I(M) \leq 2\left|N_{G} K / K\right|$. Since the automorphisms $\Phi_{1}^{\sigma}$ are induced by the free action of $N_{G} K / K$ on $S U_{n} / K$, we have $L\left(\Phi_{1}^{\sigma}\right)=0$ for all $\sigma \neq$ id. For $\lambda=-1$, we consider the limit in 5.1 as $\lambda \rightarrow-1$. This limit will be zero unless there are as many factors $1-\lambda^{i}, i$ even, in the denominator as there are in the numerator. There are [ $n / 2]$ such factors in the numerator, where brackets indicate the greatest integer function, while the denominator has

$$
\begin{aligned}
& \sum_{\left|S_{p}\right| \text { even }} n\left(S_{p}\right)+\sum_{\left|S_{p}\right| \text { odd }}\left[\frac{n\left(S_{p}\right)}{2}\right] \\
& =\sum_{\left|S_{p}\right| \text { even }} \sum_{i \in S_{p}} \frac{n_{i}}{\left|S_{p}\right|}+\sum_{\left|S_{p}\right| \text { odd }} \sum_{i \in S_{p}} \frac{1}{\left|S_{p}\right|}\left[\frac{n_{i}}{2}\right] .
\end{aligned}
$$


Since the $n_{i}$ sum to $n$, (4) can equal [ $n / 2$ ] only if $\left|S_{p}\right| \leq 2$ for all $p$ and, with perhaps one exception if $n$ is odd, $n\left(S_{p}\right)$ is even whenever $\left|S_{p}\right|=1$. Since each set $S_{p}$ must be contained in some $M_{q}$, there exists a $\sigma$ whose partition $\left\{S_{p}\right\}$ satisfies these conditions only if at most one of $\left\{m_{q} \mid q\right.$ odd $\}$ is odd. In this case, there exists $\sigma$ with $\Phi_{-1}^{\sigma} \neq 0$, and we can use Proposition 2.6 (with $R=\left\{\mathrm{id}, \Phi_{-1}^{\sigma}\right\}$ ) to conclude that $R N I(M) \leq\left|N_{G} K / K\right|$.

To complete the proof we need only show that $R N I(M) \geq 2\left|N_{G} K / K\right|$ in the case where two or more of $\left\{m_{q} \mid q\right.$ odd $\}$ are odd: we do this by explicit construction. First we define a map $J: \mathbf{C}^{n} \rightarrow \mathbf{C}^{n}$ as follows. If $n=2 p$ is even, regard $\mathbf{C}^{n}$ as the quaternionic vector space $\mathbf{H}^{p}$ and define $J$ to be multiplication by the quaternion $j$; if $n=2 p+1$ is odd, think of $\mathbf{C}^{n}$ as $\mathbf{H}^{p} \oplus \mathbf{C}$ and let $J(v, z)=(j v, \bar{z})$. Then $J$ is a conjugate-linear automorphism of $\mathbf{C}^{n}$ that preserves orthogonality, and $\mathbf{C}^{n}$ cannot have two orthogonal $J$-invariant subspaces of odd dimension (cf. Theorem 1 of [6]). Now regard $S U_{n} / K$ as the space of decompositions of form (1), and define $2\left|N_{G} K / K\right|$ self-maps of $S U_{n} / K$ by

$$
\begin{aligned}
\left(p_{1}, \ldots, p_{k}\right) \rightarrow\left(p_{\sigma(1)}, \ldots, p_{\sigma(k)}\right), & \sigma \in N_{G} K / K \\
\left(p_{1}, \ldots, p_{k}\right) \rightarrow\left(J p_{\sigma(1)}, \ldots, J p_{\sigma(k)}\right), & \sigma \in N_{G} K / K .
\end{aligned}
$$

Clearly there are no coincidences among the maps in (5) or those in (6). Now suppose there is a coincidence between a map in (5) and one in (6): then there is an element $\left(p_{1}, \ldots, p_{k}\right)$ of $S U_{n} / K$ with

$$
p_{\sigma(i)}=J p_{\pi(i)}, \quad 1 \leq i \leq k,
$$

for some $\sigma, \pi \in N_{G} K / K$. Then the subspaces

$$
\bigoplus_{n_{1}=i} p_{j}, \quad m_{i} \neq 0,
$$

are $J$-invariant and have dimension $i m_{i}$. Since at least two of $m_{1}$, $m_{3}, \ldots$ are odd, this contradicts the properties of $J$. The conclusion then follows.

REMARK. If $n$ is even, $J$ has period 2 as a self-map of $S U_{n} / K$, and the group $N_{G} K / K \times \mathrm{Z}_{2}$ acts on $S U_{n} / K$ via

$$
(\sigma, \varepsilon) \cdot\left(p_{1}, \ldots, p_{k}\right)=\left(J^{\varepsilon} p_{\sigma(1)}, \ldots, J^{\varepsilon} p_{\sigma(k)}\right) .
$$

By the argument above, this action is free if two or more of $m_{1}, m_{3}, \ldots$ are odd. It is in general not orientation-preserving, however, since 
$(\sigma, \varepsilon)$ has degree $(-1)^{\varepsilon d} \operatorname{sgn} \hat{\sigma}$, where

$$
d=\sum_{i<j} n_{i} n_{j}
$$

is the complex dimension of $S U_{n} / K$ and $\hat{\sigma}$ is the restriction of $\sigma$ to $\left\{i \mid n_{i}\right.$ is odd $\}$ (as follows from Theorem 2.3 of [12]).

If $n_{1}=1, H^{*}\left(S U_{n} / K ; \mathbf{Q}\right)$ may have nontrivial endomorphisms that factor through the cohomology of $\mathrm{CP}^{n-1}$. These 'projective' endomorphisms are classified in [5] as follows. Let

$$
1-t^{n}=P_{1}(t) P_{2}(t) \cdots P_{k}(t)
$$

be a factorization in $\mathbf{Z}[t]$ with $P_{i}(t)$ a polynomial of degree $n_{i}$ and $P_{r}(t)=1-t$ for some $r$ with $n_{r}=1$. Then

$$
\Psi\left(1+x_{i 1}+\cdots+x_{i n_{i}}\right)=P_{i}\left(-\lambda x_{r 1}\right), \quad 1 \leq i \leq k,
$$

defines a projective endomorphism $\Psi$, and all projective endomorphisms have this form. We call $\lambda$ the multiplier of $\Psi$.

THEOREM 5.3. Let $M=S U_{n} / K$ be a complex flag manifold with no cohomology endomorphisms except permutation automorphisms and projective endomorphisms. Then $N I(M)=R N I(M)$ unless $k=3$, $n_{1}=n_{2}=1$, and $n_{3}$ is even.

Proof. It is shown in [10] that $S U_{n} / K$ has noncoincidence index $\infty$ when $k=3, n_{1}=n_{2}=1$, and $n_{3}$ is even, so we can exclude this case. In view of the preceding result, it is enough to show that $L Z\left(S U_{n} / K\right)$ contains no projective endomorphisms when $k>2$. Now Theorem 3 of [7] requires that any projective endomorphism of $H^{*}\left(S U_{n} / K\right.$; $\left.\mathbf{Q}\right)$ induced by a self-map have multiplier divisible by 2 or 3 . But Proposition 5 of [6] says that a projective endomorphism of Lefschetz number zero has multiplier -1 .

At present there is no known flag manifold $S U_{n} / K$ that fails to satisfy the hypothesis of Theorem 5.3. It seems reasonable to conjecture that this is always the case, but the existing evidence is somewhat limited. Full classifications of the endomorphisms of $H^{*}\left(S U_{n} / K ; \mathbf{Q}\right)$ are available only in the following cases:

1. $k=2$ and $n_{1} \leq 3$ or $n_{2} \geq 2 n_{1}^{2}-n_{1}-1$ [4];

2. $k=3, n_{1}=1$, and $n_{3} \geq 2 n_{2}^{2}-1$ [5];

3. $n_{1}=n_{2}=\cdots=n_{k-1}=1[\mathbf{1 0}]$.

In cases covered by $1-3$ we can use Theorem 5.3 to conclude that $N I\left(S U_{n} / K\right)=R N I\left(S U_{n} / K\right)$, except when $k=3, n_{1}=n_{2}=1$, and $n_{3}$ 
is even. Theorem 1.4 now follows from 1.2 and the remark following 5.2 , except in the latter case. In this case there are fixed-point-free selfmaps of degree zero, but they do not preserve the subring of $N_{G} K / K$ invariants in $H^{*}\left(S U_{n} / K\right.$; Q) (cf. Theorem 4.1 of [10]). Thus the orbit space has the fixed point property by 3.1 .

\section{REFERENCES}

[1] E. Fadell, On a coincidence theorem of F. B. Fuller, Pacific J. Math., 15 (1965), 825-834.

[2] Recent results in the fixed point theory of continuous maps, Bull. Amer. Math. Soc., 76 (1970), 10-29.

[3] F. B. Fuller, The existence of periodic points, Ann. of Math., (2) 57 (1953), 229-230.

[4] H. Glover and W. Homer, Endomorphisms of the cohomology rings of finite Grassmann manifolds, Lecture Notes in Math., vol. 657, Springer-Verlag, New York, 1978, pp. 179-183.

[5] , Self-maps of flag manifolds, Trans. Amer. Math. Soc., 267 (1981), 423434.

[6] - Fixed points on flag manifolds, Pacific J. Math., 101 (1982), 303-306.

[7] H. Glover, W. Homer and R. Stong, Splitting the tangent bundle of projective space, Indiana U. Math. J., 31 (1982), 161-166.

[8] B. Halpern, Fixed points for iterates, Pacific J. Math., 25 (1968), 255-275.

[9] M. Hoffman, On fixed point free maps of the complex flag manifold, Indiana U. Math. J., 33 (1984), 249-255.

[10] _ Noncoincidence index of manifolds, Pacific J. Math., 115 (1984), 373383.

[11] Homological restrictions on free group actions, Indiana U. Math. J. (to appear).

[12] M. Hoffman and W. Homer, On cohomology automorphisms of complex flag manifolds, Proc. Amer. Math. Soc., 91 (1984), 643-648.

[13] J. M. Kister, Microbundles are fiber bundles, Annals of Math., (2) 80 (1964), 190-199.

[14] C. McGibbon, Self-maps of projective spaces, Trans. Amer. Math. Soc., 271 (1982), 325-346.

[15] J. W. Milnor, Microbundles, Part I, Topology, 3 Suppl. 1 (1964), 53-80.

[16] S. Papadima, Rigidity properties of compact Lie groups modulo maximal tori, Math. Annalen, 275 (1986), 637-652.

[17] H. Samelson, On small maps of manifolds, Pacific J. Math., 15 (1965), 14011403.

[18] H. Shiga and M. Tezuka, Cohomology automorphisms of some homogeneous spaces, Topology Appl., 25 (1987), 143-150.

Received November 5, 1987 and in revised form November 30, 1987. Research partially supported by a grant from the Naval Academy Research Council. 


\section{PACIFIC JOURNAL OF MATHEMATICS EDITORS}

\author{
V. S. VARADARAJAN \\ (Managing Editor) \\ University of California \\ Los Angeles, CA 90024 \\ HeRbert Clemens \\ University of Utah \\ Salt Lake City, UT 84112 \\ THOMAS ENRIGHT \\ University of California, San Diego \\ La Jolla, CA 92093
}

R. FINN

Stanford University

Stanford, CA 94305

HERMANN FLASCHKA

University of Arizona

Tucson, AZ 85721

VAUGHAN F. R. JONES

University of California

Berkeley, CA 94720

STEVEN KERCKHOFF

Stanford University

Stanford, CA 94305

\section{ROBION KIRBY}

University of California

Berkeley, CA 94720

C. C. MOORE

University of California

Berkeley, CA 94720

HAROLD STARK

University of California, San Diego

La Jolla, CA 92093

\section{ASSOCIATE EDITORS}
R. ARenS
E. F. BECKENBACH
B. H. NeumanN
F. WOLF
K. YOSHIDA (1906-1982)

\section{SUPPORTING INSTITUTIONS}
UNIVERSITY OF ARIZONA
UNIVERSITY OF OREGON
UNIVERSITY OF BRITISH COLUMBIA
UNIVERSITY OF SOUTHERN CALIFORNIA
CALIFORNIA INSTITUTE OF TECHNOLOGY
STANFORD UNIVERSITY
UNIVERSITY OF CALIFORNIA
MONTANA STATE UNIVERSITY
UNIVERSITY OF HAWAII
UNIVERSITY OF NEVADA, RENO
UNIVERSITY OF TOKYO
NEW MEXICO STATE UNIVERSITY
UNIVERSITY OF UTAH
OREGON STATE UNIVERSITY
WASHINGTON STATE UNIVERSITY
UNIVERSITY OF WASHINGTON 


\section{Pacific Journal of Mathematics}

\section{Vol. 136, No. $1 \quad$ November, 1989}

Robert Archbold and Frederic W. Shultz, Characterization of $C^{*}$-algebras with continuous trace by properties of their pure states $\ldots \ldots \ldots \ldots \ldots \ldots 1$

Shu Ping Chen and Roberto Triggiani, Proof of extensions of two conjectures on structural damping for elastic systems $\ldots \ldots \ldots \ldots \ldots \ldots$

Philip Throop Church and James Timourian, A nonlinear elliptic

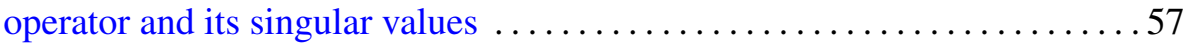

A. Gervasio Colares and Katsuei Kenmotsu, Isometric deformation of surfaces in $R^{3}$ preserving the mean curvature function $\ldots \ldots \ldots \ldots \ldots 71$

Fei Xu, A remark on spinor norms of local integral rotations. I . . . . . . . 81

Pedro Martinez Gadea and Ángel María Montesinos-Amilibia, Spaces of constant para-holomorphic sectional curvature $\ldots \ldots \ldots \ldots \ldots \ldots \ldots 5$

Guangxin Zeng, Homogeneous Stellensätze in semialgebraic geometry . . . . 103

Thomas Eric Hall, The isomorphism problem for orthodox semigroups . . . . 123

Mike Hoffman, Noncoincidence index, free group actions, and the fixed

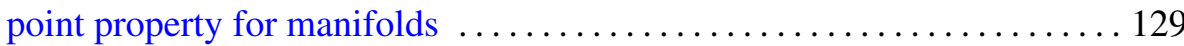

Terry Atherton Loring, The noncommutative topology of one-dimensional

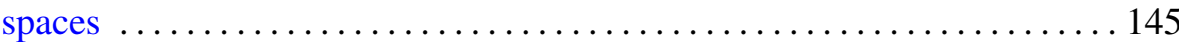

Haskell Paul Rosenthal and Alan Evan Wessel, The Krě̆ n-Mil'man property and a martingale coordinatization of certain nondentable convex sets

Yoshimi Saito, A remark on the limiting absorption principle for the reduced wave equation with two unbounded media 